\title{
SOME FINITE GROUPS WITH FEW DEFINING RELATIONS
}

\author{
B. H. NEUMANN \\ To the memory of Philip Hall, my Cambridge Doktorvater, and \\ a friend for almost half a century
}

(Received 24 October 1983)

Communicated by H. Lausch

\begin{abstract}
Some new classes of finite groups with zero deficiency presentations, that is to say presentations with as few defining relations as generators, are exhibited. The presentations require 3 generators and 3 defining relations; the groups so presented can also be generated by 2 of their elements, but it is not known whether they can be defined by 2 relations in these generators, and it is conjectured that in general they can not. The groups themselves are direct products or central products of binary polyhedral groups with cyclic groups, the order of the cyclic factor being arbitrary.
\end{abstract}

1980 Mathematics subject classification (Amer. Math. Soc.): 20 F 05.

\section{Introduction}

Let the group $\mathscr{G}$ be generated by a (finite) family $\left\{a_{1}, a_{2}, \ldots, a_{d}\right\}$ of its elements, with a (finite) family $\left\{u_{1}=v_{1}, u_{2}=v_{2}, \ldots, u_{e}=v_{e}\right\}$ of defining relations, where the $u_{i}, v_{i}$ are words in the generators $a_{1}, a_{2}, \ldots, a_{d}$. These data constitute a presentation of $\mathscr{G}$, and I write

$$
\mathscr{G}=\operatorname{gp}\left(a_{1}, a_{2}, \ldots, a_{d} ; u_{1}=v_{1}, u_{2}=v_{2}, \ldots, u_{e}=v_{e}\right) .
$$

This is a slightly revised and slightly amplified version of a paper that had been intended for the Festschrift for Philip Hall's 80th birthday: He died, aged 78 years, on 1982-12-30. The original version was semi-published in [11].

(c) 1985 Australian Mathematical Society $0263-6115 / 85 \$ A 2.00+0.00$ 
This notation, adapted from one first introduced by Phillip Hall to deal also with other algebraic systems than groups, will be varied slightly in obvious ways, such as writing $a, b, \ldots$ for the generators. The restriction to finite presentations, that is to say, presentations with both the number $d$ of generators and the number $e$ of relations finite, is not necessary, but convenient. The difference $d-e$ is the deficiency of the presentation. This is not an invariant of the group even if $e$ is chosen as small as the generating family will allow; one example is the group

$$
\operatorname{gp}\left(a, b ; a^{2} b=b a^{3}\right)
$$

of Baumslag and Solitar [1]: Graham Higman has remarked [6] that if this group is generated by $a_{1}=a^{4}$ and $b_{1}=b$, then at least two defining relations are required. Another example is the clover knot group, that is the fundamental group of the residual space of a clover knot in $\mathbb{R}^{3}$,

$$
\mathrm{gp}\left(a, b ; a^{2}=b^{3}\right)
$$

see Dunwoody and Pietrowski [4].

It is well known that the deficiency of every presentation of a finite group is non-positive. Thus finite groups with zero deficiency form a border-line class; Johnson [8] calls them interesting groups. Such groups, if nilpotent, can be generated by 3 or fewer elements; a finite nilpotent group that can be generated by 4 but not by 3 elements needs at least 5 defining relations; and until quite recently it was not known whether this bound is attained. Now Havas and Newman [5] have constructed groups of orders $2^{16}, 2^{17}, 2^{18}$, and $2^{19}$ with minimally 4 generators and 5 defining relations. The question whether there are finite non-nilpotent groups with minimally 4 generators and zero deficiency presentations is still open.

The present paper makes a modest contribution to the list of examples of finite groups with presentations of zero deficiency. It has reached its present form after a number of talks I gave in various places in Scotland, Australia, USA, Austria, Germany in 1981 and 1982, and I have much profited from the discussions and suggestions that arose out of these talks. I am particularly indebted, and grateful, to Professor John Leech, Professor A. J. van der Poorten, Dr R. T. Worley for help with the number theory I needed; to Mr W. A. Alford, Dr George Havas, Mr P. E. Kenne, and Dr M. F. Newman, who helped with some of the group-theoretical computing; and to Dr Newman and Professor H. S. M. Coxeter, who have drawn my attention to some relevant additional references.

\footnotetext{
${ }^{1}$ Statements described as "well-known" or without attribution can be found in Coxeter and Moser [3] or Johnson [8].
} 


\section{Binary polyhedral groups}

I begin with some known facts, in order to fix the notation here used.

Starting from the well known presentation

$$
\mathscr{P}(p, q, r)=\mathrm{gp}\left(a, b ; a^{p}=b^{q}=(a b)^{r}=1\right),
$$

which has deficiency -1 , one first forms

$$
\mathscr{B}(p, q, r)=\mathrm{gp}\left(a, b ; a^{p}=b^{q}=(a b)^{r}\right) .
$$

Here 1 stands both for the unit element of the groups that occur and for the natural number; and the parameters $p, q, r$ are integers. Nothing of interest is lost if one assumes that

$$
2 \leqslant|p| \leqslant|q| \leqslant|r| .
$$

It is well known that $\mathscr{P}(p, q, r)$ is finite if, and only if

$$
\frac{1}{|p|}+\frac{1}{|q|}+\frac{1}{|r|}>1
$$

the groups $\mathscr{P}(p, q, r)$ are then known as the polyhedral groups. Specifically they are

$$
\text { the dihedral groups: }|p|=|q|=2,|r| \geqslant 2 \text {, of order } 2|r| \text {, }
$$

for which I shall use the abbreviation $\mathscr{D}_{2|r|}$;

the tetrahedral group: $|p|=2,|q|=|r|=3$, of order 12 , abbreviated $\mathscr{T}_{12}$;

the cotahedral group: $|p|=2,|q|=3,|r|=4$, of order 24 , abbreviated $\mathcal{O}_{24}$;

the icosahedral group: $|p|=2,|q|=3,|r|=5$, or order 60 , abbreviated $\mathscr{F}_{60}$.

They are normally written with $p, q, r$ positive, as this loses no generality. However, for the groups $\mathscr{B}(p, q, r)$ derived from them, the fact that no generality is lost by assuming (2), while not deep, is not immediately obvious; and it is not true that (as I innocently assumed until fairly recently) no generality is lost by taking the parameters positive. Put, more generally (and temporarily only),

$$
\mathscr{B}(P, Q, R, \alpha, \beta)=\mathrm{gp}\left(a, b ; a^{P}=b^{Q}=\left(a^{\alpha} b^{\beta}\right)^{R}\right),
$$

where $P, Q, R$ are arbitrary non-zero integers and $\alpha= \pm 1, \beta= \pm 1$. If $|P|,|Q|$, $|R|$ are all different, there are 6 permutations of the set $\{|P|,|Q|,|R|\}$, and $2^{5}$ possible combinations of the signs of the 5 parameters to be considered, giving 192 presentations $\mathscr{B}( \pm p, \pm q, \pm r, \pm 1, \pm 1)$, where $\{p, q, r\}=\{|P|,|Q|,|R|\}$. However, it is an easy exercise to show that by chosing new generators

$$
a_{1}=b, \quad b_{1}=a,
$$

or

$$
a_{2}=a^{-1}, \quad b_{2}=b,
$$


or

$$
a_{3}=a b, \quad b_{3}=b,
$$

or by a concatenation of such changes, one can transform every $\mathscr{B}(P, Q, R, \alpha, \beta)$ to one of the 4 forms $\mathscr{B}(p, \pm q, \pm r,+1,+1)$, with $\{p, q, r\}=\{|P|,|Q|,|R|\}$ and, say, $0<p \leqslant q \leqslant r$. The presentations thus obtained, with the simpler notation (1), namely

$$
\mathscr{B}(p, \pm q, \pm r)=\operatorname{gp}\left(a, b ; a^{p}=b^{ \pm q}=(a b)^{ \pm r}\right),
$$

should be compared with those attributed to Threlfall $[14]^{2}$ by Coxeter and Moser $[3$, p. 68]:

$$
\langle l, m, n\rangle=\operatorname{gp}\left(a, b, c ; a^{\prime}=b^{m}=c^{n}=a b c\right) .
$$

The similarity becomes obvious when it is observed, as Coxeter and Moser [3, p. 69] do, that one can write

$$
\langle 2, m, n\rangle=\operatorname{gp}\left(b, c ; b^{m}=c^{n}=(b c)^{2}\right) .
$$

It follows that

$$
\langle 2, m, n\rangle \cong \mathscr{B}(2,-n, m) \cong \mathscr{B}(2,-m, n) ;
$$

the verification is not difficult, and omitted.

In particular the binary polyhedral groups, which are extensions of a central cyclic group of order 2 by a polyhedral group, are:

$\langle 2,2, r\rangle \cong \mathscr{B}(2,-2, r)$ of order $4|r|$, the dicyclic groups of Coxeter and Moser $\left[3\right.$, p. 7], for which I shall use the abbreviation $\mathscr{D}_{4|r|}^{*}$;

$\langle 2,3,3\rangle \cong \mathscr{B}(2,-3,3)$ of order 24 , the binary tetrahedral group, here abbreviated $\mathscr{T}_{24}^{*}$;

$\langle 2,3,4\rangle \cong \mathscr{B}(2,-3,4)$ of order 48 , the binary octahedral group, here abbreviated $\mathcal{O}_{48}^{*} ;$ and

$\langle 2,3,5\rangle \cong \mathscr{B}(2,-3,5)$ of order 120 , the binary icosahedral group, here abbreviated $\mathscr{S}_{120}^{*}$.

The other finite groups of the form $\mathscr{B}(p, \pm q, \pm r)$ are as follows:

The group $\mathscr{B}(2,2, r)$ has order $4 r(r-1)$; it can be described as the extension of a cyclic group of order $2 r(r-1)$, generated by $a b=f$, say, by a cyclic group generated by $a$, such that

$$
a^{-1} f a=f^{2 r-2} \text { and } a^{2}=f^{r} .
$$

\footnotetext{
${ }^{2}$ Actually Threlfall in [13] only asks for the order of $\langle 5,2,3\rangle$. In [14, footnote] he credits Max Dehn with the fact that the groups $\langle-3,2,3\rangle,\langle-4,2,3\rangle,\langle-5,2,3\rangle$ are also finite. Seifert [12] gives their orders; see also Coxeter [2].
} 
If $r$ is even, the group can also be described as the direct product

$$
\mathscr{B}(2,2, r) \cong \mathscr{D}_{4|r|}^{*} \times \mathscr{C}_{|r-1|},
$$

where $\mathscr{C}_{n}$, with $n>0$, stands for the cyclic group of order $n$. The group is isomorphic to Coxeter and Moser's $\langle-2,2, r\rangle$ (see [3, p. 70]).

The group $\mathscr{B}(2,3,3)$ has order 72 ; it can be described as a (necessarily split) extension of the quaternion group by a $\mathscr{C}_{9}$ that induces the automorphism of order 3 on the quaternion group; thus the subgroup of order 3 of the $\mathscr{C}_{9}$ is central in the whole group, and factoring it out one obtains the binary tetrahedral group $\mathscr{T}_{24}^{*}$. The group $\mathscr{B}(2,3,3)$ is $\langle-3,2,3\rangle$ in Coxeter and Moser $[3$, p. 70$]$ and also occurs in Seifert [12, p. 8]; it has recently been incorporated by Kenne [9] in an infinite sequence of cyclic extensions of the quaternion group with zero deficiency presentations. This group is also isomorphic with $\mathscr{B}(2,-3,-3)$.

The remaining finite groups $\mathscr{B}(p, \pm q, \pm r)$ are

$$
\begin{aligned}
\mathscr{B}(2,3,-3) & \cong \mathscr{T}_{24}^{*} \times \mathscr{C}_{7}, \\
\mathscr{B}(2,3,4) & \cong \mathscr{O}_{48}^{*} \times \mathscr{C}_{7}, \\
\mathscr{B}(2,3,-4) & \cong \mathscr{O}_{48}^{*} \times \mathscr{C}_{13}, \\
\mathscr{B}(2,-3,-4) & \cong \mathcal{O}_{48}^{*} \times \mathscr{C}_{5}, \\
\mathscr{B}(2,3,5) & \cong \mathscr{I}_{120}^{*} \times \mathscr{C}_{19}, \\
\mathscr{B}(2,3,-5) & \cong \mathscr{I}_{120}^{*} \times \mathscr{C}_{31}, \\
\mathscr{B}(2,-3,-5) & \cong \mathscr{I}_{120}^{*} \times \mathscr{C}_{11} .
\end{aligned}
$$

All these groups were determined by Coxeter [2], and most of them are listed in Coxeter and Moser [3, p. 70]. The last three occur already in Hölder [7, pp. 350 sqq.], with rather different presentations. Note that here, as in Coxeter [2], all these groups have zero deficiency presentations. In the next section infinite sequences of such groups with zero deficiency presentations will be constructed, of which the above groups are special cases.

\section{New presentations}

Building on the groups considered in the preceding section, I now introduce groups with the following presentations:

$$
\mathscr{G}(p, q, r, s, t, u)=\operatorname{gp}\left(a, b, c ; a^{p}=c^{s}, b^{q}=c^{t},(a b)^{r}=c^{u}\right),
$$

where $p, q, r, s, t, u$ are integral parameters, which will presently be restricted so as to make the groups finite. Nothing of interest is lost if one assumes that

$$
0<|p| \leqslant|q| \leqslant|r|
$$


and as no restrictions will be placed on the signs of $s, t, u$, I shall even assume, without loss of generality, that

$$
0<p \leqslant q \leqslant r .
$$

The case $p=1$ is somewhat exceptional and will be treated later; for the present $I$ shall, therefore, further assume

$$
2 \leqslant p \leqslant q \leqslant r .
$$

It is clear that putting $c=1$ will give $\mathscr{P}(p, q, r)$ as a factor group of $\mathscr{G}(p, q, r, s, t, u)$; thus it is natural to restrict attention to the polyhedral cases

$$
\frac{1}{p}+\frac{1}{q}+\frac{1}{r}>1 \text {. }
$$

Equally necessary to ensure finiteness of $\mathscr{G}=\mathscr{G}(p, q, r, s, t, u)$ is that the factor group of the derived group, $\mathscr{G} / \mathscr{G}^{\prime}$, should be finite. This requires that the matrix

$$
\left(\begin{array}{lll}
p & 0 & -s \\
0 & q & -t \\
r & r & -u
\end{array}\right)
$$

be non-singular, that is that the determinant is non-zero:

$$
\Delta=-p q u+p r t+q r s \neq 0 .
$$

In the presence of the inequality (3) and the inequation (4), a sufficient condition for finiteness of $\mathscr{G}$ is that $s, t, u$ are pairwise coprime. Less is necessary when $p=1$, vide infra, and when $p=q=2$; but when $p=2, q=3, r=3$ or 4 or 5 , this coprimality appears to be necessary, too.

\section{LEMMA 1. If}

$$
(s, t)=(s, u)=(t, u)=1,
$$

then $c$ is central in $\mathscr{G}(p, q, r, s, t, u)$.

Proof. As $c^{s}$ is a power of $a$, it commutes with $a$; similarly $c^{u}$ commutes with $a b$. Thus $c^{s u}$ commutes with both $a$ and $a b$, hence with $b$. But also $c^{t}$ commutes with $b$, and as $(s u, t)=1$, then also $c$ commutes with $b$. Then $c^{u}$ commutes with both $a b$ and $b$, hence with $a$, and as $(s, u)=1$, then $c$ also commutes with $a$.

THEOREM 2. Conditions (3), (4), (5) are jointly sufficient to ensure the finiteness of $\mathscr{G}(p, q, r, s, t, u)$.

Proof. Condition (5) ensures that $c$ is central in $\mathscr{G}=\mathscr{G}(p, q, r, s, t, u)$, and condition (4) ensures that a non-trivial power of $c$, for example $c^{\Delta}$, falls into the derived group $\mathscr{G}^{\prime}$. Let $\kappa$ be the order of $c$ modulo $\mathscr{G}^{\prime}$. Then $c^{\kappa}$ lies in both the 
centre and the derived group of $\mathscr{G}$, hence in the Schur multiplicator of $\mathscr{G}^{*}$, the factor group of $\mathscr{G}$ modulo the (cyclic normal) subgroup generated by $c^{\kappa}$. Now $\mathscr{G}^{*}$ is a finite group, because it is an extension of the central subgroup generated by the image $c^{*}$ of $c$, of order $\kappa$, by an isomorphic copy of the polyhedral group $\mathscr{P}(p, q, r)$-which is finite because of condition (3). But the multiplicator of a finite group is finite; hence $c^{\kappa}$ has finite order-in the cases under consideration the order is, in fact, 2 . It follows that $\mathscr{G}$ is finite, and in fact an extension of a central cyclic group of order 2 by $\mathscr{G}^{*}$.

To compute the order of $\mathscr{G}$, it is first necessary to compute $\kappa$. Now if the index of the derived group $\mathscr{P}^{\prime}$ of the polyhedral group $\mathscr{P}=\mathscr{P}(p, q, r)$ is denoted by $\Gamma$, then clearly

$$
|\Delta|=\kappa \Gamma,
$$

where $\Delta$ is given by (4). The values of $\Gamma$ are well known; they are:

for the dihedral groups $\mathscr{D}_{2 r}$ :

if $r$ is odd, $\Gamma=2$;

if $r$ is even, $\Gamma=4$;

for the tetrahedral group $\mathscr{T}_{12}: \Gamma=3$;

for the octahedral group $\mathcal{O}_{24}: \Gamma=2$;

for the icosahedral group $\mathscr{I}_{60}: \Gamma=1$.

The order of $\mathscr{G}$ is then simply

$$
|\mathscr{G}|=2 \kappa|\mathscr{P}|=2|\mathscr{P}| \frac{|\Delta|}{\Gamma} .
$$

Thus the order of $\mathscr{G}$ depends on the particular polyhedral group involved and on $|\Delta|$; and $\Delta$ depends linearly on $s, t, u$, say

$$
\Delta=k_{1} s+l_{1} t+m_{1} u
$$

where $k_{1}=q r, l_{1}=p r, m_{1}=-p q$. The coefficients $k_{1}, l_{1}, m_{1}$ here are all divisible by $\Gamma$, say $k_{1}=\Gamma k, l_{1}=\Gamma l, m_{1}=\Gamma m$, so that

$$
\frac{\Delta}{\Gamma}=k s+l t+m u \text {. }
$$

The actual coefficients that arise from the polyhedral cases are as follows:

$$
\begin{aligned}
& k=r, \quad l=r, \quad m=-2, \quad(\text { dihedral with odd } r) ; \\
& k=\frac{1}{2} r, \quad l=\frac{1}{2} r, \quad m=-1 \quad \text { (dihedral with even } r \text { ); } \\
& k=3, \quad l=2 \quad m=-2 \quad \text { (tetrahedral); } \\
& k=6, \quad l=4, \quad m=-3 \quad \text { (octahedral); } \\
& k=15, \quad l=10 \quad, m=-6 \quad \text { (icosahedral). }
\end{aligned}
$$

Thus in all cases $(k, l, m)=1$. 
To determine the possible values of $|\Delta| / \Gamma$, and thus the orders that the groups $\mathscr{G}$ can have, one has to find all values of $n$ for which the linear diophantine equation

$$
k s+l t+m u=n
$$

has a solution $s, t, u$ subject to

$$
(s, t)=(s, u)=(t, u)=1,
$$

given that the coefficients $k, l, m$ are jointly coprime:

$$
(k, l, m)=1 \text {. }
$$

In the polyhedral cases one can show, by ad hoc arguments, that the equation (6) subject to (5) has solutions for every $n$; and I am grateful to Professor John Leech for providing me with an elegant such solution in the icosahedral case. But it would be more satisfactory to establish the following proposition:

THEOREM 3. Let $k, l, m, n$ be integers with $(k, l, m)=1$ and $n$ arbitrary; then there are infinitely many integer solutions of the diophantine equation

$$
k s+l t+m u=n
$$

subject to $(s, t)=(s, u)=(t, u)=1$.

This is indeed true, and using a suggestion of Professor A. J. van der Poorten I managed to prove it (and its natural generalization to more than 3 variables), employing the heavy guns of Dirichlet's theorem on primes in arithmetic progressions. However, recently R. T. Worley [15] has found a straightforward proof of the (generalised) theorem:

WORLEY's THEOREM. If $k_{1}, k_{2}, \ldots, k_{d}$ are integers such that $\left(k_{1}, k_{2}, \ldots, k_{d}\right)=1$, then for each integer $n$ the equation

$$
k_{1} x_{1}+k_{2} x_{2}+\cdots+k_{d} x_{d}=n
$$

subject to

$$
\left(x_{i}, x_{j}\right)=1 \text { for } 1 \leqslant i<j \leqslant d
$$

has infinitely many solutions.

\section{Interlude}

The case $p=1$ will be briefly considered. The groups $\mathscr{P}(1, q, r)$ and $\mathscr{B}(1, q, r)$ are obviously cyclic, and their orders are easily determined. The structure of $\mathscr{G}(1, q, r, s, t, u)$ is less obvious. Replacing the generator $a$ by $c^{s}$ and omitting it 
from the presentation, one has

$$
\mathscr{G}=\mathscr{G}(1, q, r, s, t, u)=\mathrm{gp}\left(b, c ; b^{q}=c^{t},\left(c^{s} b\right)^{r}=c^{u}\right) .
$$

As before, a necessary condition for finiteness of $\mathscr{G}$ is that the matrix

$$
\left(\begin{array}{cc}
q & -t \\
r & r s-u
\end{array}\right)
$$

be non-singular, that is that the determinant be non-zero:

$$
\Delta=q r s-q u+r t \neq 0 .
$$

This is, of course, the special case $p=1$ of condition (4). It does not suffice to ensure finiteness of $\mathscr{G}$; for example, $\mathscr{G}(1,2,2,2,2,2)$, with $\Delta=8$, is an extension of a central group of order 2 by the infinite dihedral group. By contrast, $\mathscr{G}(1,2,2,1,2,2)$ is the quaternion group. The verification of these facts is easy, and omitted.

THEOREM 4. Condition (7) jointly with

$$
(t, u)=1
$$

is sufficient to ensure that $\mathscr{G}(1, q, r, s, t, u)$ is a finite cyclic group, of order $|\Delta|$.

Proof. As $c^{t}$ is a power of $b$, it commutes with $b$; similarly $c^{u}$ commutes with $c^{s} b$, and thus also with $b$. As $t$ and $u$ are coprime, it follows that $c$ commutes with $b$, and the group is abelian; it has order $|\Delta|$ because this is the index in it of its derived group, now seen to be the trivial group. Finally a finite abelian group with a zero deficiency presentation, and hence with trivial Schur multiplicator, must be cyclic.

Thus, for example,

$$
\mathrm{gp}\left(b, c ; b^{4}=c^{-7},\left(c^{4} b\right)^{5}=c^{11}\right)
$$

is a presentation of the trivial group; is this trivial? I think not, though a bare-handed proof is not too difficult. In fact, P. E. Kenne, using a computer program he has devised [9] to prove such facts from an analysis of the computer execution of a Todd-Coxeter enumeration has produced a proof in 9 steps. The Todd-Coxeter coset enumeration, not the one Mr. Kenne employed, but one using a procedure due to Felsch, had to generate more than 2,800 cosets to show the group to be trivial-which it did in very few seconds!

There are other conditions that ensure that the group is abelian and thus, under assumption of (7), finite cyclic; for example, if $(q, r)=1$ and $t$ divides $s$, one shows by an equally easy argument that the group is abelian. 


\section{Further remarks}

It has been shown that the groups $\mathscr{G}=\mathscr{G}(p, q, r, s, t, u)$ are, for the "polyhedral" choices of $p, q, r$ and for suitable choices of $s, t, u$, extensions of a cyclic central subgroup $\mathscr{C}_{2 n}$ of arbitrarily prescribed even order $2 n$ by the polyhedral group that belongs to the choice of $p, q, r$. In many cases, for example if $n$ is not divisible by 2 or 3 , or if $p=2, q=3, r=5$, that is in the icosahedral case, $\mathscr{G}$ is the central product of $\mathscr{C}_{2 n}$ and the corresponding binary polyhedral group, amalgamating the central involutions; but some different groups also turn up, for example the group of order 72 described as $\mathscr{B}(2,3,3)$ in Section 2, which here occurs as $\mathscr{G}(2,3,3,1,1,1)$. [In fact all groups $\mathscr{B}(p, \pm q, \pm r)$ occur as $\mathscr{G}(p, q, r, 1, \pm 1, \pm 1)$.]

All the groups here considered can be generated by two elements; for if $\sigma, \tau$ are so chosen that

then

$$
\sigma s+\tau t=1
$$

$$
a^{p \sigma} b^{q \tau}=c ;
$$

thus $c$ is superfluous as a generator. Do the groups then also have a zero deficiency presentation when generated by $a$ and $b$ ? In some cases they have, for example if $p=q=2$ (the dihedral case); but in general I do not know the answer, and I conjecture that in general the answer is negative. In particular I conjecture that if $\mathscr{G}(2,3,5, s, t, u)$ is generated by $a, b$ only, then for all sufficiently large values of

$$
\Delta=15 s+10 t-6 u
$$

the group requires 3 defining relations. If this is true, then it provides examples of finite groups with presentations that, even in terms of the least number of defining relations, have different deficiencies. However, while single relation presentations of groups have been studied in great depth, starting with the fundamental paper [10] of Wilhelm Magnus, (and it is this that allows one to verify Higman's remark about the Baumslag and Solitar group mentioned in the Introduction), far too little seems to be known about presentations with more than one relation.

\section{References}

[1] Gilbert Baumslag and Donald Solitar, 'Some two-generator one-relator non-Hopfian groups', Bull. Amer. Math. Soc. 68 (1962), 199-201.

[2] H. S. M. Coxeter, 'The binary polyhedral groups, and other generalizations of the quaternion group', Duke Math.J. 7 (1940), 367-379. 
[3] H. S. M. Coxeter and W. O. J. Moser, Generators and relations for discrete groups, Fourth edition [Ergebnisse der Mathematik und ihrer Grenzgebiete 14], Springer-Verlag, Berlin, 1980.

[4] M. J. Dunwoody and A. Pietrowski, 'Presentations of the trefoil groups', Canad. Math. Bull. 16 (1973), 517-520.

[5] George Havas and M. F. Newman, 'Minimal presentations for finite groups of prime-power order', Comm. Algebra 11 (1983), 2267-2275.

[6] Graham Higman (unpublished).

[7] Otto Hölder, 'Bildung zusammengesetzter Gruppen', Math. Ann. 46 (1895), 321-422.

[8] D. L. Johnson, Topics in the theory of group presentations, [London Math. Soc. Lecture Note Series 42], Cambridge University Press, Cambridge, \&c, 1980.

[9] Peter Emil Kenne, PhD thesis, Australian National University, Canberra, 1984.

[10] Wilhelm Magnus, 'Über diskontinuierliche Gruppen mit einer definierenden Relation. (Der Freiheitssatz),' $J$, reine angew. Mathematik 163 (1930), 141-165.

[11] B. H. Neumann, 'Some finite groups with few defining relations', Mathematics Research Report No. 40. The Australian National University, 1982. $14 \mathrm{pp}$.

[12] H. Seifert, 'Zweite Lösung der Aufgabe 84', Jber. Deutsche Math. Verein. 41 (1932), 7-8.

[13] W. Threlfall, 'Aufgave 84, Jber. Deutsche Math. Verein. 39 (1930), 86.

[14] W. Threlfall, 'Erste Lösung der Aufgabe 84', Jber. Deutsche Math. Verein. 41 (1932), 6-7.

[15] R. T. Worley, 'Pairwise relatively prime solutions of linear diophantine equations', J. Austral. Math. Soc. Ser. A 37 (1984), 39-44.

Department of Mathematics

Institute of Advanced Studies

Australian National University

Canberra, ACT 2601

Australia

and

Division of Mathematics and Statistics

Commonwealth Scientific and

Industrial Research Organization

Canberra, ACT 2601

Australia 